\title{
Großräumige Regionalisierung der Kohlenstoffbindung in Wäldern Nordrhein-Westfalens
}

\author{
Winfried Schröder • Gunther Schmidt • Roland Pesch
}

Eingegangen: 1. April 2009/Akzeptiert: 23. August 2009/Online veröffentlicht: 17. September 2009

(C) Springer-Verlag 2009

Zusammenfassung Hintergrund, Ziel und Bereich Die Klimarahmenkonvention der Vereinten Nationen sieht in der $\mathrm{CO}_{2}$-Bindung des Waldes einen wichtigen Beitrag zur Reduktion klimarelevanter Treibhausgase. Bezogen auf die gesamte Waldfläche Deutschlands wird die durchschnittliche Speicherung von Kohlenstoff (C) auf 214t C/ha geschätzt. Eine räumliche Differenzierung dieses Schätzwertes liegt nicht vor. In der vorgestellten Untersuchung sollte ein Konzept für die großräumige Differenzierung solcher Durchschnittwerte am Beispiel von Nordrhein-Westfalen (NRW) auf der Grundlage von landes- bzw. bundesweiten Inventurdaten und digitalen Karten entwickelt und erprobt werden. Dieses Verfahren soll einen Beitrag für das Klimabiomonitoring liefern.

Material und Methoden Zu diesem Zweck wurden Daten der Landeswaldinventur (LWI) und der Bodenzustandserhebung (BZE) in Verbindung mit flächenhaft vorliegenden Geoinformationen zusammengeführt und mit geostatistischen und entscheidungsbaumanalytischen Verfahren ausgewertet. Die geostatistischen Methoden (Variografie und Kriging) ließen sich für die C-Pools der Humusschicht nutzen. Für die C-Vorräte in der oberirdischen Biomasse und im Mineralboden wurde ein auf den Entscheidungsbaumverfahren Classification and Regression Trees (CART) und Chi-Square Automatic-Interaction Detection Method (CHAID) basierender prädiktiver Ansatz gewählt.

Ergebnisse Für die Wälder in NRW konnten im oberirdischen Derb- und Totholz ca. 59Mio. Tonnen Kohlenstoff

W. Schröder · G. Schmidt $\cdot$ R. Pesch $(\bowtie)$

Lehrstuhl für Landschaftsökologie, Hochschule Vechta,

Postfach 1553, 49364 Vechta, Deutschland

E-Mail: rpesch@iuw.uni-vechta.de

W. Schröder

E-Mail: wschroeder@iuw.uni-vechta.de
(67 t C/ha), für die Humusauflage ca. 17 Mio. Tonnen Kohlenstoff (19,3 t C/ha) und für den Mineralboden (bis max. $2 \mathrm{~m}$ Tiefe) ca. 80 Mio. Tonnen Kohlenstoff (90,7 t C/ha) berechnet und räumlich differenziert werden. Daraus ergibt sich für die Wälder Nordrhein-Westfalens ein Kohlenstoffvorrat von ca. 156 Mio. Tonnen C für diese drei Kompartimente. Dies entspricht einem durchschnittlichen Wert von ca. $177 \mathrm{t} \mathrm{C/ha}$.

Diskussion In anderen Untersuchungen wurden ähnliche, allerdings räumlich nicht differenzierte Durchschnittswerte berechnet: Demnach sind in den Wäldern von NRW in der oberirdischen Dendromasse, die sich allerdings neben dem in diese Studie eingeflossenen Derb- und Totholz auch aus dem Reisig der Bäume zusammensetzt, ca. 68 Mio. Tonnen Kohlenstoff gespeichert. Der durchschnittliche Kohlenstoffgehalt der Humusauflage der Waldfläche Deutschlands wird auf 20,7 t C/ha geschätzt. Damit liegt dieser bundesdeutsche Durchschnittswert etwas höher als in der Humusauflage der nordrhein-westfälischen Wälder. In den Wäldern Thüringens sind durchschnittlich $27,7 \pm 8,1 \mathrm{t} \mathrm{C}$ /ha gebunden, unter Nadelwald 34,0 t/ha und unter Laubwald 11,3t/ha. Für den Mineralboden bis zu einer maximalen Tiefe von $90 \mathrm{~cm}$ wird für die Waldfläche Deutschlands ein Durchschnittswert von 87,9 t $\mathrm{C} /$ ha geschätzt. Dieser Wert liegt nahe bei dem in dieser Studie ermittelten Wert von 90,7t C/ha. Die Kohlenstoffgehalte im Mineralboden weisen je nach Ausgangsgestein, Genese und Wasserhaushalt der Böden große Unterschiede auf: Böden aus kalkhaltigem Ausgangsgestein haben mit bis zu 115t/ha die höchsten C-Gehalte, während für stauwassergeprägte Pseudogleye mit $47 \mathrm{t} /$ ha die niedrigsten C-Gehalte ermittelt wurden.

Schlussfolgerungen Die Flächenberechnungen zu den Kohlenstoffvorräten in der oberirdischen Biomasse, in der Humusauflage und im Mineralboden ermöglichen einen landesweiten Überblick der räumlichen Differenzierung der Kohlenstoffgehalte von Wäldern sowie der Randbedingun- 
gen für die Fixierung des klimarelevanten Treibhausgases Kohlendioxid in Waldökosystemen Nordrhein-Westfalens.

Empfehlungen und Ausblick Der entwickelte Ansatz sollte anhand bundesweit vorliegender Daten und durch Anwendung des Regression Kriging validiert werden. Zusätzlich sollte berechnet werden, wie sich die Kohlenstoffspeicherung unter veränderten Klimabedingungen entwickeln wird. Hierfür sollten sowohl statistische Verfahren als auch dynamische Modellierungen, z. B. mit dem Modellsystem WASMOD, eingesetzt werden.

Schlüsselwörter C-Fixierung $\cdot$ C-Sequestrierung · C-Speicherung · Forstökosysteme $\cdot$ Geostatistik · CART (Classification and Regression Trees) $\cdot$ CHAID (Chi-Square Automatic Interaction Detection) · GIS · Klimabiomonitoring $\cdot$ Regionalisierung $\cdot$ Treibhausgase

\section{Regionalising the carbon fixation in forests of North Rhine-Westphalia using inventory data and digital maps}

\begin{abstract}
Background, aim and scope The United Nations Framework Convention on Climate Change understands carbon fixation in forests as an important contribution for the reduction of atmospheric pollution in terms of greenhouse gases. According to the German forest inventory on carbon in biomass an amount of $191 \mathrm{t} \mathrm{C/ha}$ was roughly estimated, without any spatial differentiation. Therefore, the aim of this investigation was to statistically identify factors that are significant for the carbon fixation and to map the spatial patterns of $\mathrm{C}$ sequestration in the federal state North Rhine-Westphalia.

Materials and methods Together with information on climate, elevation, vegetation, and deposition, data from two forest monitoring networks were analysed statistically. Geostatistics and the decision tree algorithms Classification and Regression Trees (CART) and Chi-Square Automatic Interaction Detection (CHAID) were applied to calculate surface maps from punctual data on $\mathrm{C}$ in vegetation, in dead wood and in soil. Whereas spatial autocorrelation could be detected for the $\mathrm{C}$ loads in the humus layers, no surface maps could be calculated for the $\mathrm{C}$ contents of the mineral soils and for the forest trees/dead wood. Here, CART and CHAID were used to derive decision trees that were applied on available surface data to predict $\mathrm{C}$ loads for the entire study area.

Results About $19 \mathrm{t} \mathrm{C/ha}$ could be predicted for the humus layer, $67 \mathrm{t} \mathrm{C/ha}$ for forest trees/dead wood and $90.7 \mathrm{t} \mathrm{C} / \mathrm{ha}$ for the soil. An overall mean of $177 \mathrm{t} \mathrm{C} /$ ha was calculated for North Rhine-Westphalia lying $14 \mathrm{t} \mathrm{C/ha}$ below the German wide mean.
\end{abstract}

Discussion Compared to the calculated results in another investigation a total of $68 \mathrm{mio}$. $\mathrm{t} \mathrm{C}$ for the above ground den- dromass was estimated. This is $11 \mathrm{mio} . \mathrm{t} \mathrm{C} /$ ha higher than the amount calculated in this study and may be due to the fact that this value includes the C-pools in both, the brushwood and herbaceous layer in their estimations. The average $\mathrm{C}$ concentration in the humus layer all over Germany was found to amount for $20.7 \mathrm{t} \mathrm{C} /$ ha which is slightly above the $\mathrm{C}$ storage calculated for North Rhine-Westphalia. In the same study a Germany wide $\mathrm{C}$ average of $87.9 \mathrm{t} \mathrm{C} /$ ha was calculated which is very close to the $90.7 \mathrm{t} \mathrm{C} /$ ha calculated in this study.

Conclusions The surface estimations of the C-pools in the above-ground biomass, the humus layer and the mineral soil enable to map the efficiency of the $\mathrm{C}$-bounding capacity regarding the fixation of the greenhouse gas $\mathrm{CO}_{2}$. The mean values derived in this study are in good accordance with estimations based on other techniques.

Recommendations and perspectives The approach presented should be verified by application to Germany wide inventory data and by means of Regression Kriging. Furthermore, the C-fixation under climate change should be calculated by combining statistical methods and the dynamic modelling tool WASMOD.

Keywords Biomonitoring of climate change $\cdot \mathrm{C}$-fixation C-pools $\cdot \mathrm{C}$-sequestration $\cdot \mathrm{C}$-storage $\cdot$ Geostatistics $\cdot$ CART (Classification and Regression Trees) $\cdot$ CHAID (Chi-Square Automatic Interaction Detection) $\cdot$ Forest ecosystems $\cdot$ GIS $\cdot$ Regionalisation

\section{Hintergrund, Ziel und Bereich}

Die globale Durchschnittstemperatur der erdnahen Atmosphäre ist zwischen 1906 und 2005 um $0,74^{\circ} \mathrm{C}\left( \pm 0,18^{\circ} \mathrm{C}\right)$ angestiegen. Elf der vergangenen zwölf Jahre zwischen 1995 und 2006 gehörten zu den zwölf wärmsten seit Beginn der Temperaturmessungen 1850. Der Trend der vergangenen 50 Jahre liegt mit einer gemessenen Erwärmung um $0,13^{\circ} \mathrm{C}\left( \pm 0,03^{\circ} \mathrm{C}\right)$ pro Jahrzehnt nahezu doppelt so hoch wie für die vergangenen 100 Jahre. In den vergangenen 100 Jahren stieg die Temperatur bei gleichzeitig zunehmender Variabilität durchschnittlich um $0,95^{\circ} \mathrm{C}$ in Europa, und von 1990 bis 2100 ist mit weiteren $2,0^{\circ} \mathrm{C}$ bis $6,3^{\circ} \mathrm{C} \mathrm{zu}$ rechnen (EEA 2004). In Deutschland nahm die Lufttemperatur von 1991 bis 2002 um durchschnittlich $0,8^{\circ} \mathrm{C}$ und regional um bis $\mathrm{zu} 3,5^{\circ} \mathrm{C} \mathrm{zu}$ (Schröder et al. 2007). Der vierte Sachstandsbericht des Zwischenstaatlichen Ausschusses für Klimaänderungen (Intergovernmental Panel on Climate Change - IPCC) der Vereinten Nationen ist der jüngste Bericht in einer Reihe, die regelmäßig den wissenschaftlichen Kenntnisstand über die globale Erwärmung zusammenfasst. Demnach wird eine weitere Erderwärmung erwartet (IPCC 2007). Wie hoch diese sein könnte, hängt von Annahmen über die Bevölkerungsentwicklung oder das Wirtschafts- 
wachstum ab: Im Vergleich zur globalen Durchschnittstemperatur der letzten vollständigen Klimanormalperiode 1961 bis 1990 wird demnach der mittlere Anstieg der weltweiten Durchschnittstemperatur bis zur Dekade 2090 bis 2100 im günstigsten angenommenen Szenario auf $1,8^{\circ} \mathrm{C}$ bei einer möglichen Bandbreite von 1,1 bis $2,9^{\circ} \mathrm{C}$ geschätzt, im ungünstigsten Szenario auf $4,0^{\circ} \mathrm{C}$ bei einer denkbaren Streubreite von 2,4 bis $6,4^{\circ} \mathrm{C}$.

Der Klimawandel ist nicht auf rein meteorologische Phänomene beschränkt, sondern er wirkt breit auf alle von der Sonnenenergie angetriebenen Vorgänge in Ökosystemen (Beinhauer 1999). Solche Auswirkungen des Klimawandels sind vorzugsweise anhand biologischer Indikatoren fassbar (IPCC 2002, 2007). Böden und Wälder sind Gegenstände des Biomonitoring (Zierdt 1997). Sie sind für die Modellierung der Klimaveränderungen, ihrer Folgen und für Anpassungsmaßnahmen von Bedeutung. Die Klimarahmenkonvention der Vereinten Nationen sieht in der $\mathrm{CO}_{2}$-Bindung des Waldes einen wichtigen Beitrag zur Reduktion klimarelevanter Treibhausgase. Zwar speichern die Wälder mit weltweit 2060 Gt C relativ wenig Kohlenstoff (C) im Vergleich zu den Ozeanen (38.000 Gt C). Doch ist der C-Speicher Wald durch die Landnutzung (Ausweitung der Waldflächen) und die Wirtschaftsweise (Baumartenwahl) beeinflussbar. Mit 2 Gt pro Jahr entspricht das C-Flusspotenzial der Wälder dem der Ozeane und übertrifft das der Atmosphäre um 1 Gt C (Joosten und Schulte 2001).

In Nordrhein-Westfalen (NRW) werden auf etwa 880.000 ha Waldfläche schätzungsweise 68 Mio. Tonnen Kohlenstoff in der oberirdischen Dendromasse (Derb- und Totholz, Reisig) gespeichert (Joosten und Schulte 2001). Hierbei zeigen sich baumartenspezifische Unterschiede und eine Reihenfolge, die von der zweiten Altersklasse an konstant bleibt: Buche $>$ Fichte $>$ Eiche $>$ Kiefer. Obwohl die Buche in NRW einen Waldflächenanteil von 16,5\% hat, speichert sie 24,7\% der oberirdischen C-Menge. Die C-Gehalte des Derb- und Totholzes, des Reisigs, der Blätter und
Nadeln, der Wurzeln, der Strauch- und Krautschicht, der Humusauflage und des Mineralbodens werden zusammen auf $209 \mathrm{t} C$ pro ha geschätzt. Eine räumliche Differenzierung der C-Vorräte war bislang nicht möglich, und für eingehende ökologische Interpretationen der C-Vorräte fehlen eine synthetisierende, multivariat-statistische Auswertungen der Inventurdaten (BZE, LWI) über die C-Vorräte in den Böden und in der oberirdischen Biomasse sowie der Daten über relevante Einflussfaktoren (u. a. Relief, Geologie, Klima, Bodenprofilmorphologie und Bodenchemie). Daher war es das Ziel dieser Untersuchung, die C-Vorräte in der Biomasse und in den Böden der Wälder von NRW unter Berücksichtigung der Klima-, Standort- und Bestandesdaten flächenhaft differenzierend zu berechnen und in einem geografischen Informationssystem (GIS) zu kartieren. Die Studie wurde im EU Forest Focus Programm (Freer-Smith et al. 2004) im Auftrag des Landesamtes für Natur, Umwelt und Verbraucherschutz Nordrhein-Westfalen durchgeführt.

\section{Material und Methoden}

In Abschn. 2.1 und 2.2 wird ein Überblick der verwendeten Daten und Methoden vermittelt. Diese Angaben werden zum besseren Verständnis der Ergebnisse durch detailliertere Angaben zu den Daten und zur Ergebnisberechnung ergänzt (Kap. 3).

\subsection{Daten}

Für diese Untersuchung wurden die in Tabelle 1 zusammengestellten Daten verwendet. Im Folgenden werden die Daten der Landeswaldinventur (LWI) von NRW aus dem Jahr 1998 und die zwischen 1987 und 1993 erhobenen Daten der bundesweiten Bodenzustandserhebung (BZE I) näher beschrieben. Die LWI ist eine terrestrische Stichprobenerhebung mit einem systematischen $1 \mathrm{~km} \times 1 \mathrm{~km}$-Raster über das

Tabelle 1 Datengrundlagen (LANUV Landesamt Landesamt für Natur, Umwelt und Verbraucherschutz NRW)

\begin{tabular}{|c|c|c|c|c|c|}
\hline Name & Beschreibung & Auflösung/Maßstab & Format & Quelle & Erhebung \\
\hline LWI & Landeswaldinventur NRW & 8788 Standorte & Punkt & LANUV & 1998 \\
\hline BZE & Bodenzustandserhebung & 498 Standorte & Punkt & LANUV & 1989-1991 \\
\hline ATKIS-Wald-Layer & Waldverbreitung & $1: 25.000$ & Polygon & LANUV & 2001 \\
\hline BK 50 NRW & Bodenübersichtskarte & $1: 50.000$ & Polygon & LANUV & 2001 \\
\hline $\begin{array}{l}\text { Klima (Lufttemperatur, } \\
\text { Niederschlag, Verdunstung) }\end{array}$ & $\begin{array}{l}\text { Mittelwerte und Summen der Klima- } \\
\text { merkmale (Grid für LWI, Polygon für } \\
\text { BZE) }\end{array}$ & $1 \mathrm{~km} \times 1 \mathrm{~km}$ & Grid, Polygon & $\begin{array}{l}\text { DWD, Klima-Atlas } \\
\text { NRW }\end{array}$ & $\begin{array}{l}1961-1990 \\
1951-1980\end{array}$ \\
\hline Naturräume & $\begin{array}{l}\text { Haupteinheiten der naturräumlichen } \\
\text { Gliederung }\end{array}$ & $1: 1.000 .000$ & Polygon & $\begin{array}{l}\text { Meynen et al. } \\
(1953-1962)\end{array}$ & $1943-1962$ \\
\hline Raumgliederung & $\begin{array}{l}\text { Ökologische Raumgliederung } \\
\text { Deutschland }\end{array}$ & $2 \mathrm{~km} \times 2 \mathrm{~km}$ & Grid & Schröder et al. (2006) & 2002 \\
\hline Wuchsgebiete/Wuchsbezirke & Forstgliederung NRW & $1: 1.000 .000$ & Polygon & LANUV & 2002 \\
\hline
\end{tabular}


gesamte Gebiet von NRW. Bei einer Waldfläche von rund 880.000 ha ergeben sich 8788 Inventur-Standorte. Hauptziel der LWI ist es, eine Mengenabschätzung des Holzbestandes und mögliche Holzvorratveränderungen des Waldes bis hinunter auf die lokale Ebene eines Forstbezirkes belegen zu können. Zu diesem Zweck wird die Masse des Derbund Totholzes in konzentrischen Kreisen eines jeden Rasterpunktes ermittelt. Das Derbholzvolumen des Standortes wird aus der Holzmasse mit einer Stärke von mindestens $7 \mathrm{~cm}$ im Querschnitt abgeschätzt. Ebenso wird beim stehenden Totholz verfahren. Beim liegenden Totholz werden nur die Stücke miteinbezogen, deren dünneres Ende $>20 \mathrm{~cm}$ aufweist und die mindestens $2 \mathrm{~m}$ lang sind. $\mathrm{Zu}$ den für die Massenermittlung benötigten Primärdaten zählen Baumart, Brusthöhendurchmesser (BHD), Kronenansatz, Baumhöhe und die Güteklasse des unteren Stammabschnitts. Aus diesen Daten wird zunächst durch Multiplikation des Einzelbaumvolumens mit der baumartenspezifischen Raumdichte nach Knigge und Schulz (1966) die Holztrockenmasse je Einzelbaum hergeleitet. Durch Multiplikation mit der Gewichtung und durch Hochrechnung ergeben sich die Holzmassen je Hektar. Der C-Vorrat je Standort wird pauschal mit $50 \%$ der Holzmasse angesetzt.

Das Ziel der bundesweiten BZE ist es, quantitative Angaben über den Zustand und mögliche Veränderungen des Waldbodens zu erhalten. Die erste BZE des Landes NRW wurde in den Jahren 1989 bis 1991 auf den Level-I-Standorten (Waldzustandserhebung, WZE) in einer räumlichen Auflösung von $4 \mathrm{~km} \times 4 \mathrm{~km}$ durchgeführt. Landesweit sind somit rund 500 Standorte bodenkundlich aufgenommen worden. Die Bodenproben wurden aus verschiedenen Tiefenstufen (bis max. $2 \mathrm{~m}$ Tiefe) als Mischproben aus neun Bohrungen von jedem Inventurpunkt gewonnen. Neben weiteren chemischen Kenngrößen kann so auch der C-Gehalt des Bodens hinreichend genau beziffert werden (BMELF 1994). Die Standorte der BZE befinden sich jeweils etwa 200 bis $400 \mathrm{~m}$ südwestlich der LWI-Erfassungsflächen.

Um eine Regionalisierung der punktuell vorliegenden oberirdisch im Derb- und Totholz sowie der in Mineralboden und Humusauflage gebundenen C-Vorräte zu ermöglichen, wurden weitere flächenhaft vorhandene Informationen von NRW eingebunden. Hierzu gehören die Karte der forstlichen Wuchsgebiete/-bezirke (AKS 2005), die Bodenkarte im Maßstab 1:50.000 und Klimadaten des Deutschen Wetterdienstes. Die geografische Verteilung der Waldflächen wurde dem Amtlich topografisch-kartografischen Informationssystem (ATKIS) entnommen.

\subsection{Methoden}

Die flächenhafte Differenzierung der C-Vorräte in der Biomasse und im Boden wurde durch geostatistische Methoden und Verfahren des Data Mining aus den in Abschn. 2.1 be- schriebenen Daten abgeleitet. Die von Fränzle und Killisch (1980) in die Umweltwissenschaften eingeführten geostatistischen Methoden basieren auf der Theorie der regionalisierten Variablen (Matheron 1965). Dabei wird ein räumlich verorteter Messwert als die Realisierung einer dort vorliegenden Wahrscheinlichkeitsfunktion angesehen und angenommen, dass diese Realisierungen in einer räumlichen Abhängigkeit zueinander stehen. Räumlich abhängig bedeutet, dass sich Messwerte dicht beieinander liegender Standorte ähnlicher sind als Messwerte weiter entfernt liegender Standorte. Diese auch als räumliche Autokorrelation bezeichnete Annahme wird mit der Variogrammanalyse überprüft. Dabei wird die räumliche Korrelation der Daten mit einem (Semi-)Variogramm untersucht. Ein Variogramm zeigt die halbe gemittelte quadrierte Differenz zwischen allen Messwertpaaren innerhalb einer Abstandsklasse. Deren Größe sollte nicht größer sein als die Hälfte des maximalen Abstandes zwischen den Messpunkten (je Richtung) im Untersuchungsgebiet.

Dem experimentellen Variogramm wird eine Modellkurve angepasst. Zur Berechnung jedes Variogrammwertes sollten etwa 30Datenpaare zur Verfügung stehen. Der ansteigende Teil des Modellvariogramms sollte auf mindestens drei bis vier Variogrammwerten beruhen. Das Variogramm steigt aufgrund des autokorrelativen Zusammenhangs, der über die Entfernung in der Regel abnimmt, meist im Ursprung steil an und flacht dann ab einer bestimmten Entfernung ab. Der Abstand, bei dem das Variogramm asymptotisch den Schwellenwert (sill) erreicht, wird Reichweite (range) genannt. Diese gibt an, bis zu welchem Abstand räumliche Korrelationen auftreten und welche Punkte zur Kriging Interpolation genutzt werden sollten. Schwellenwert und Reichweite können sich richtungsabhängig unterscheiden. Anisotropie ist also dann gegeben, wenn die Daten in unterschiedliche Richtungen unterschiedlich autokorreliert sind. Der AnisotropieFaktor gibt das Verhältnis der Reichweiten unterschiedlicher Richtungen zueinander an (Webster und Oliver 2001).

Das Variogramm müsste durch den Ursprung verlaufen, denn die räumliche Varianz eines Punktes mit sich selbst ist Null. Verläuft das Variogramm nicht durch den Ursprung, dann existiert ein sogenannter Nuggeteffekt, der dem Abstand von 0 bis zum Schnittpunkt des Variogramms mit der $y$-Achse entspricht. Dieser Effekt kann auf Messfehlern oder auf nicht erfassten Mikrovariabilitäten beruhen. Die Bezeichnung Nuggeteffekt geht auf die Analyse von Goldlagerstätten in Südafrika zurück, in denen Proben, die sehr eng beieinander liegen, hohe Goldgehalte (nugget) oder kein Gold enthalten können. Dominiert der Nuggeteffekt die räumliche Variabilität, dann ist der Mittelwert der beste Schätzer. Mit zunehmendem Nuggeteffekt verlieren die nahe liegenden Messpunkte an Bedeutung, die weiter entfernt liegenden erhalten höhere Gewichte. Als Daumenregel gilt, dass der Nuggeteffekt max. die Hälfte der Semivarianz ausmachen, besser aber unter einem Drittel derselben 
liegen sollte (Webster and Oliver 2001). Dennoch kann es durchaus sinnvoll sein, auch bei höheren Nuggeteffekten Kriging-Prozeduren durchzuführen. Die derart geschätzten räumlichen Strukturen erscheinen stark geglättet und weniger differenziert. Großräumige Unterschiede sind aber dennoch auszumachen.

Das experimentelle Variogramm beschreibt die räumliche Struktur einer Messgröße. Bei der Interpolation mit Kriging (Krige 1951) wird es zur Bestimmung der Schätzgewichte benötigt. Da Schätzgewichte für alle Abstände - auch für nicht in den Daten vorhandene - benötigt werden, muss eine Modellfunktion an die berechneten Variogrammwerte angepasst werden. Die Schätzung erfolgt beim Kriging mit einer gewichteten linearen Kombination benachbarter Messwerte. Neben der Bestimmung des Schätzwertes wird die Schätzvarianz berechnet. Die Schätzvarianz gibt Informationen über die Zuverlässigkeit der Schätzung: Je kleiner die Schätzvarianz, desto besser das Ergebnis. Die Schätzwerte sollen erwartungstreu sein. Die Differenzen zwischen den geschätzten und den gemessenen Werten sollen im Mittel Null sein (Webster und Oliver 2001).

Neben den Ausprägungen von Nugget, Sill und Schätzvarianz sind statistische Kennzahlen der Kreuzvalidierung wichtig für die Bewertung der Qualität der Kriging-Interpolation (Isaaks und Srivastava 1989). Bei der Kreuzvalidierung (Cross Validation) wird aus der Menge aller Wertepunkte nach und nach jeweils ein Punkt entfernt und dann mit allen restlichen die Kriging-Interpolation durchgeführt. Der nicht in die Interpolation einfließende Punkt dient als Prüfgröße. Er enthält den wahren Wert eines Phänomens an einem bestimmten Ort. Auf dieselbe Weise wird jeweils ein anderer Punkt aus der Menge der Punkte entfernt und mit der gewählten Methode und deren Parametern interpoliert. Am Ende der durchgeführten Prozedur wurde jeder Datenpunkt einmal nicht in die Interpolation einbezogen. Für jeden Punkt existiert dann ein Schätzwert, den man dem tatsächlich gemessenen Wert gegenüber stellt. Diejenige Kombination aus Variogrammmodell und Kriging-Variante, die die geringsten Differenzen zwischen den tatsächlich gemessenen und den geschätzten Werten aufweisen, ist die am besten geeignete.

Neben den geostatistischen Verfahren wurden zur Regionalisierung der Kohlenstoffvorräte in Wäldern NordrheinWestfalens Data-Mining-Techniken eingesetzt. Diese dienen der Strukturentdeckung in komplexen Datenmatrizen. Hierfür wurden die von Schröder und Schmidt (2001) bzw. Schröder (1989) in die Umweltwissenschaften eingeführten Entscheidungsbaumalgorithmen CART (Classification and Regression Trees) (Breimann et al. 1984) und CHAID (Chisquared Automatic Interaction Detection) (Kass 1980) eingesetzt. Die LWI- und BZE-Datensätze sind Matrizen, die aus den Merkmalsträgern (hier die Standorte der LWI und BZE) und denen ihnen zugeordneten Merkmalen (Boden-, Relief-, Klima-, Standort- und Bestandeseigenschaften) und ihren Merkmalen bestehen. In den CART- und CHAID-Berechnungen fungieren die C-Vorräte als Zielvariable (abhängige Variable), deren fachlich plausiblen Randbedingungen als Prädiktoren (Deskriptoren, beschreibende Variablen) genutzt werden. Mit CART und CHAID wurden statistische Beziehungen zwischen der Zielvariablen und den Prädiktoren ermittelt und als hierarchisch gestufte Entscheidungsregeln in Form von Dendrogrammen (Entscheidungsbäumen) dargestellt. Die Berechnung eines Entscheidungsbaums beruht auf der schrittweisen binären Partitionierung des Ausgangsdatensatzes (auch Wurzelknoten, root node, parent node) in Untergruppen (auch Unterknoten, sub nodes, child nodes, daughter nodes).

CHAID ermöglicht die gleichzeitige Verarbeitung ordinaler und nominaler Daten. Die jeweiligen Ausgangsmengen werden schrittweise in disjunkte Klassen aufgeteilt. Hierzu wird geprüft, welcher Prädiktor die Stichprobe anhand seiner Ausprägungsstufen am deutlichsten in signifikant voneinander verschiedene Untergruppen gliedert. Dabei wird untersucht, ob die Zusammenlegung von Stufen des Prädiktors die Trennschärfe erhöht. CHAID quantifiziert den Zusammenhang zwischen dem Kriterium und einem Prädiktor anhand des $p$-Levels durch eine Sequenz von Zusammenfassungen und Zerlegungen, die durch geeignete Assoziationsanalysen gesteuert werden. Der Prädiktor mit dem kleinsten $p$-Level im Assoziationstest wird zur Segmentierung eines Knotens herangezogen. Das $p$-Level wird auch als Irrtumswahrscheinlichkeit bezeichnet. Der Wert des $p$-Levels kann zwischen 0 und 1 liegen. Je näher der $p$-Level bei 0 liegt, umso besser ist das Ergebnis zu bewerten. Abhängig vom Skalenniveau des Kriteriums werden die $p$-Levels folgendermaßen berechnet: Bei einem metrischen Kriterium kommt der F-Test zum Einsatz, ordinale Kriterien werden mit einem sog. Y-Verknüpfungsmodell angepasst und per Likelihood-Quotienten-Test beurteilt; handelt es sich um ein nominales Kriterium, so wird der $\chi^{2}$-Test mit Pearson oder Liklihood-Quotienten-Prüfgröße verwendet (Baltes-Götz 2004).

Bei CART wird die Aufteilung nicht wie bei CHAID durch die empirischen Irrtumswahrscheinlichkeiten von Assoziationstests gesteuert, sondern über ein zu minimierendes Inhomogenitätsmaß, welches wiederum in Abhängigkeit vom Skalenniveau des Kriteriums ausgewählt wird: Für nominale Daten stehen die Inhomogenitätsmaße Gini und Twoing zur Auswahl, für ordinale Daten wird das ordinale Twoing und für metrische Daten die Least Square Deviation angewendet. Zur binären Zerlegung eines Knotens wird derjenige Prädiktor gesucht, welcher den stärksten Abfall der Inhomogenität bewirkt. Mit CART können sowohl nominale als auch kontinuierliche metrische Daten verarbeitet werden. Dies gilt sowohl für die Zielvariable als auch für die Prädiktoren.

Die hierarchisch strukturierten CART- und CHAIDRegelsysteme können auf Geodatenbanken angewendet 
werden, die Informationen über die Prädiktoren enthalten. Liegen also Daten über alle oder einige der als relevant detektierten Prädiktoren des Kohlenstoffvorrats flächenhaft für die gesamte Waldfläche des Landes Nordrhein-Westfalen vor, kann durch Anwendung der Regeln durch eine entsprechende Datenbankabfrage eine räumlich differenzierte Schätzung der Kohlenstoffvorräte und ihre prädiktive Kartierung erfolgen (Pesch et al. 2008). Entscheidende Bedeutung für die Verfahren CART und CHAID kommt daher der Auswahl der Prädiktoren zu. Die Güte des Modells ist also in erster Linie davon abhängig, ob die räumliche Verteilung der Kohlenstoffvorräte mit den beschreibenden Variablen hinreichend erklärt werden kann.

\section{Ergebnisse}

\subsection{Abschätzung der Kohlenstoffvorräte} in der Humusauflage

Eine flächenhafte Kriging-Schätzung der Kohlenstoffvorräte konnte für die Humusauflage der BZE-Standorte be-

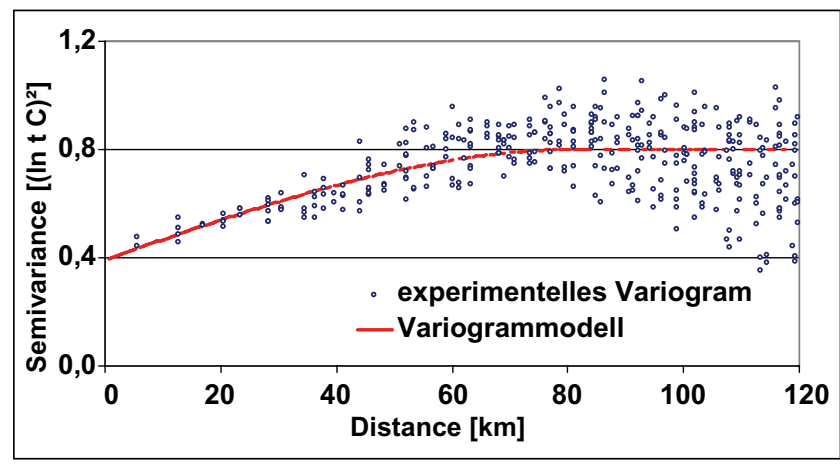

Abb. 1 Modellvariogramm für die C-Vorräte in der Humusauflage der BZE-Standorte Nordrhein-Westfalens

rechnet werden. Die dafür notwendige räumliche Autokorrelation der C-Gehalte konnte nachgewiesen und modelliert werden (Abb. 1).

Abbildung 2 zeigt das Ergebnis der Lognormal-Kriging-Flächenschätzung. Demzufolge befinden sich im östlichen Teil des Sauerlandes und im Westmünsterland die höchsten Kohlenstoffgehalte im Humus. Dies ist auf
Abb. 2 Kriging-Flächenschätzungen der C-Vorräte in der Humusauflage nordrhein-westfälischer Wälder

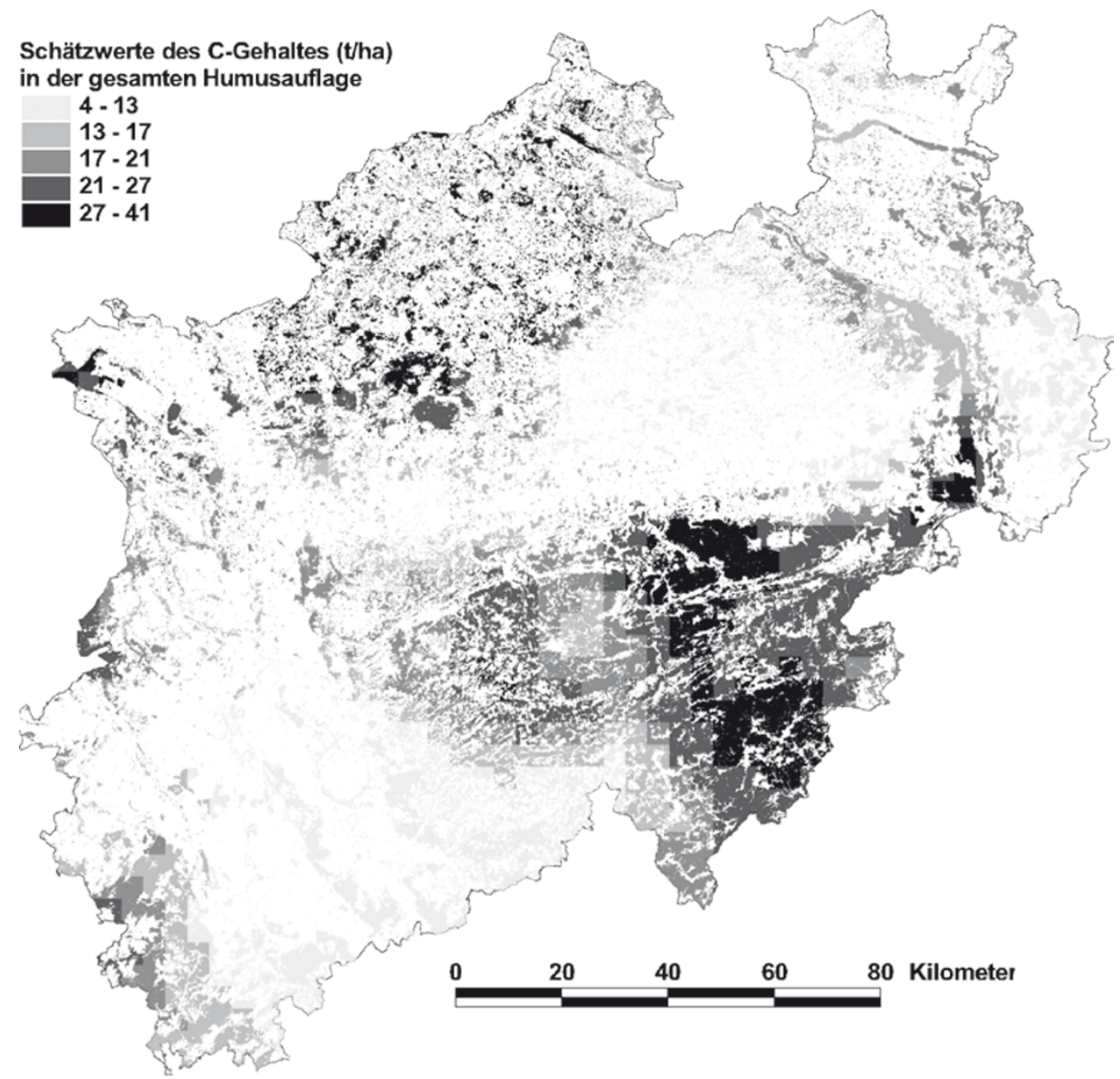


den hohen Anteil an Nadelbäumen (Fichte und Kiefer) in diesen Gebieten zurückzuführen. Die Menge an Kohlenstoff in der Humusauflage der Wälder von NRW kann mit ca. 17 Mio. $t$ angegeben werden. Das entspricht ca. 19,3 t C/ha.

Die Kreuzvalidierung ermöglicht eine Einschätzung der Güte der Flächenschätzung. So konnte eine mittlere prozentuale Abweichung zwischen den Mess- und den aus der Kreuzvaliderung resultierenden Schätzwerten von rund $35 \%$ festgestellt werden. Die Ergebnisse verweisen des Weiteren auf keine tendenziellen Über- und Unterschätzungen der verwendeten Variogrammmodelle.

\subsection{Abschätzung der Kohlenstoffvorräte im Mineralboden}

Da die Kohlenstoffvorräte im Mineralboden keine räumliche Autokorrelation aufwiesen, konnte kein Modellvariogramm angepasst werden. Eine Flächenschätzung über das Kriging-Verfahren war somit nicht möglich. Aus diesem Grunde wurde das CHAID-Verfahren auf den Datensatz der C-Gehalte im Mineralboden der BZE-Standorte angewendet. Folgende Prädiktoren wurden dabei benutzt:

Gelände: Höhe ü. NN, Reliefform, Neigungsstufe und Exposition.

Forstliche Situation: Vorbestandsgeschichte, Bestandesstruktur, Mischungsform, Schlussgrad, Düngung, Hauptbaumart, Oberhöhe und Wuchsleistung der Hauptbaumart sowie ökologische Standortfeuchte.

Boden: Bodentyp, Humusform, biologische Zustandsform, Bodenart, Basenversorgung, Grundwasser- bzw. Staunässestufe und nutzbare Feldkapazität.

Klima: Niederschlagssumme pro Jahr und pro Vegetationsperiode, Mitteltemperatur der Luft im Jahr und in der
Vegetationsperiode, Anzahl der Tage mit einer Lufttemperatur $>5^{\circ} \mathrm{C}$ bzw. $>10^{\circ} \mathrm{C}$ und Verdunstungsrate.

Des Weiteren wurde eine Verschneidung der BZE-Standorte mit der Karte der forstlichen Wuchsbezirke durchgeführt. Die Wuchsbezirke erwiesen sich im Hinblick auf die räumliche Verteilung der Kohlenstoffvorräte im Mineralboden als wichtiger Prädiktor. Die Gesamtheit aller BZEStandorte wurde anhand dieses Prädiktors in vier Segmente eingeteilt (Abb. 3). Die Übertragung der Entscheidungsregeln auf die flächenhaft vorliegenden Prädiktoren ermöglicht die landesweite Einschätzung der C-Vorräte in den Mineralböden Nordrhein-Westfalens. Durchschnittlich ist in den Mineralböden der Wälder von NRW etwa 90,7 t/ha Kohlenstoff gebunden. Daraus ergeben sich knapp 80 Mio.t C für diesen Raum.

\subsection{Abschätzung der Kohlenstoffvorräte in der Biomasse}

Das in Abschn. 2.2 beschriebene CART-Verfahren wurde zur Abschätzung der Kohlenstoffvorräte in der Derbholzbiomasse der LWI-Standorte eingesetzt. Im Gegensatz zu den Standorten der BZE liegt hier kein derartig umfangreicher Datensatz zugrunde. Somit ist auch die Anzahl der Prädiktoren geringer. Insbesondere werden an den LWIStandorten keine bodenkundlichen Erhebungen durchgeführt. Aus diesem Grunde wurde eine Verschneidung der LWI-Standorte mit der digitalen Bodenkarte von NRW im Maßstab 1:50000 vorgenommen. Damit konnten folgende Prädiktoren für die Klassifikation mit dem CART-Verfahren in den Datensatz einfließen:

Gelände: Höhe ü. NN, Exposition und Hangneigung.

Boden: Bodentyp, Bodenart, Bodenwertzahl, Mächtigkeit des Mineralbodens, nutzbare Feldkapazität, Kationen-
Abb. 3 CHAID-Baum zur
prädiktiven Kartierung der
C-Vorräte im Mineralboden
nordrhein-westfälischer Wälder

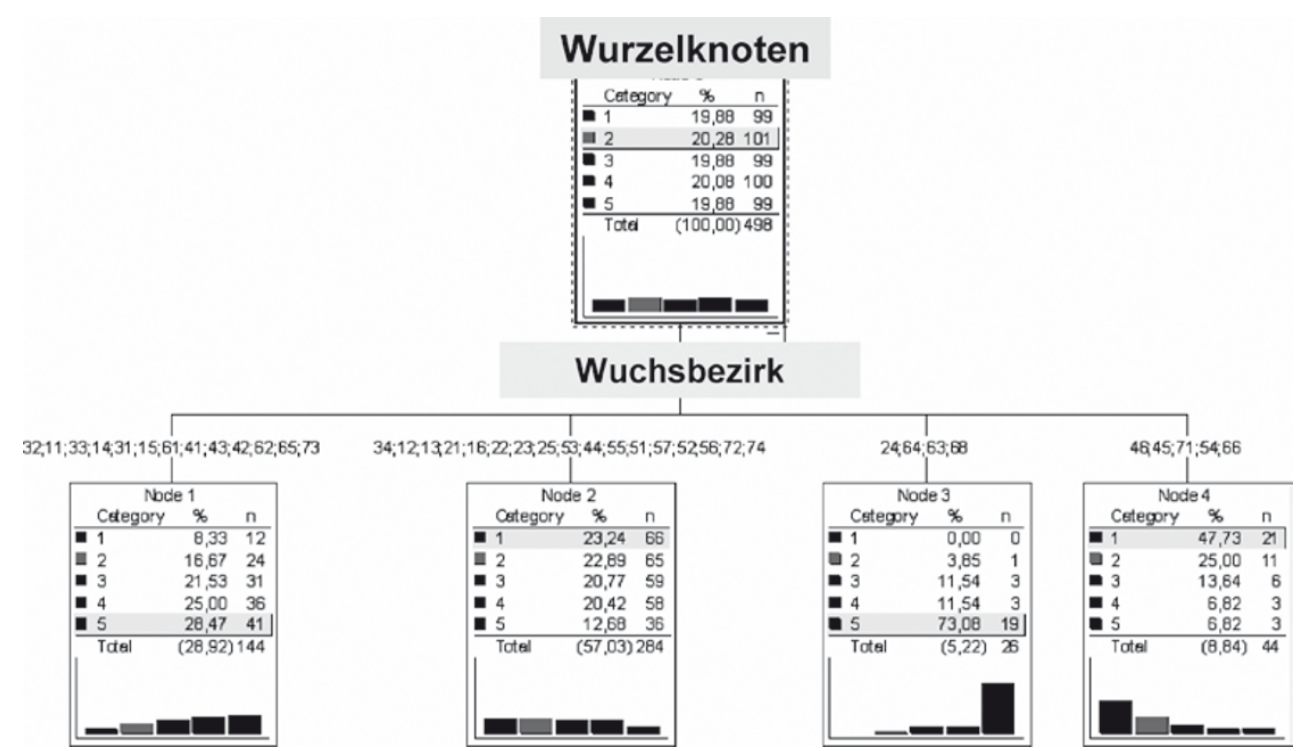




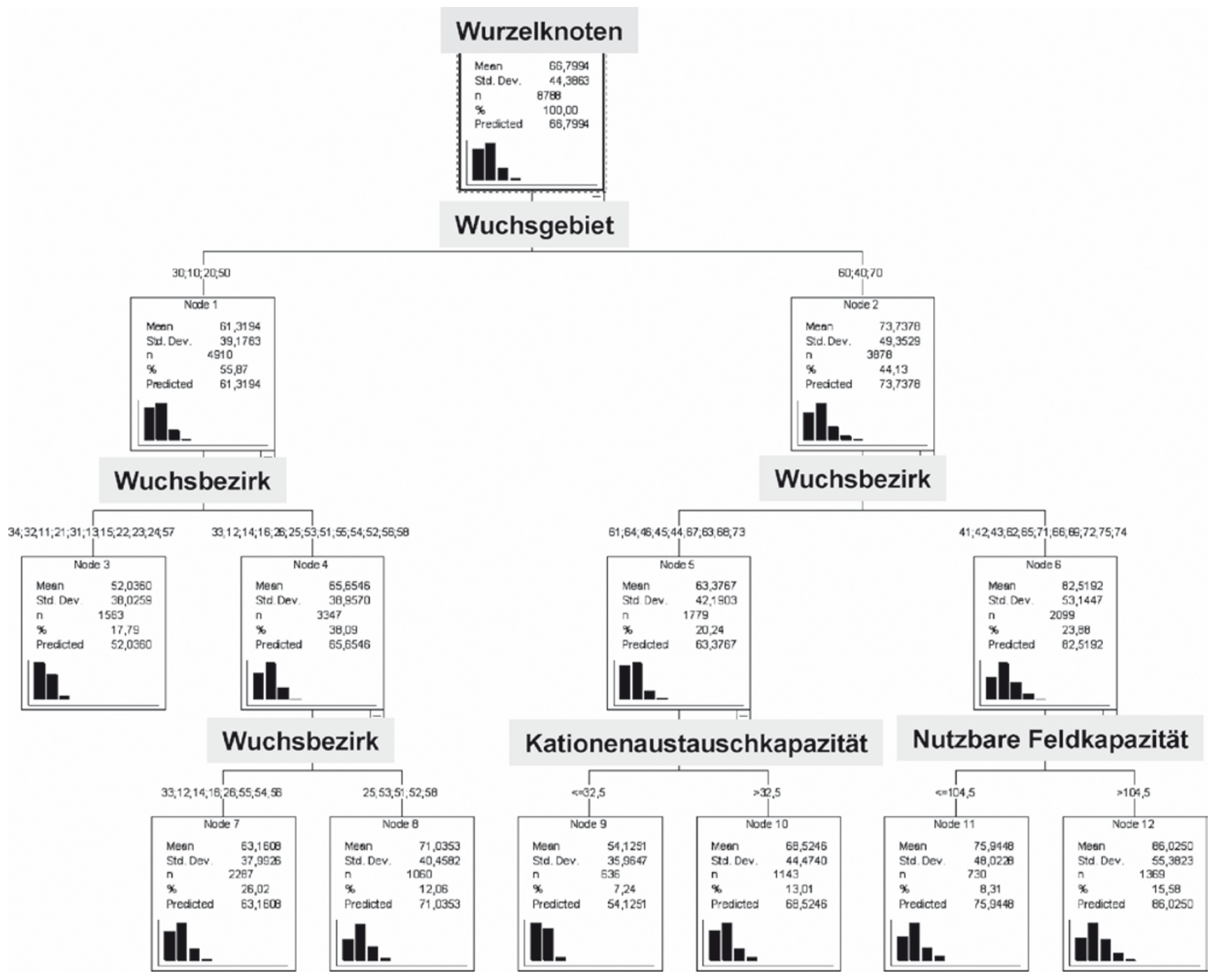

Abb. 4 CART-Baum zur prädiktiven Kartierung der Kohlenstoffvorräte in der Biomasse nordrhein-westfälischer Wälder

austauschkapazität, Wasserdurchlässigkeit und ökologische Standortfeuchte (alle Kenngrößen aus der BK 50 abgeleitet).

Klima: Niederschlagssumme pro Jahr und pro Vegetationsperiode, Mitteltemperatur der Luft im Jahr und in der Vegetationsperiode und Verdunstungsrate im Jahr und in der Vegetationsperiode.

Auch hier wurde eine Verschneidung der LWI-Standorte mit der Karte der forstlichen Wuchsbezirke durchgeführt. Als wichtigste Prädiktoren erweisen sich auch in diesem Falle die Wuchsbezirke und darüber hinaus die Bodenkenngrößen Kationenaustauschkapazität und nutzbare Feldkapazität. Es ergeben sich sieben Standortklassen (Abb. 4). Durch flächenhafte Übertragung der Entscheidungsregeln konnten folgende Werte für den Kohlenstoff in der Derbholzbiomasse der Wälder ermittelt werden: Insgesamt sind knapp 59 Mio. t $\mathrm{C}$ gespei- chert. Daraus ergibt sich ein mittlerer Gehalt von etwa $69 \mathrm{t} / \mathrm{ha}$.

3.4 Gesamtabschätzung der Kohlenstoffvorräte für den Wald

Durch Addition der in den Abschn. 3.1 bis 3.3 vorgestellten Ergebnisse ergeben sich die in Abb. 5 dargestellten Kohlenstoffvorräte für den Wald von Nordrhein-Westfalen. Demzufolge sind etwa 156Mio. t Kohlenstoff fixiert. Dies entspricht einem durchschnittlichen Wert von $177 \mathrm{t} / \mathrm{ha}$. Das relativ dicht bewaldete Bergische Land und große Teile der Westfälischen Bucht mit einem geringeren Waldflächenanteil weisen die höchsten durchschnittlichen Kohlenstoffvorräte auf. Unterdurchschnittliche Kohlenstoffmengen sind hingegen in großen Teilen des dicht bewaldeten Sauerlandes und der Nordeifel zu verzeichnen. 
Abb. 5 Gesamtabschätzung der Kohlenstoffvorräte in den nordrhein-westfälischen Wäldern

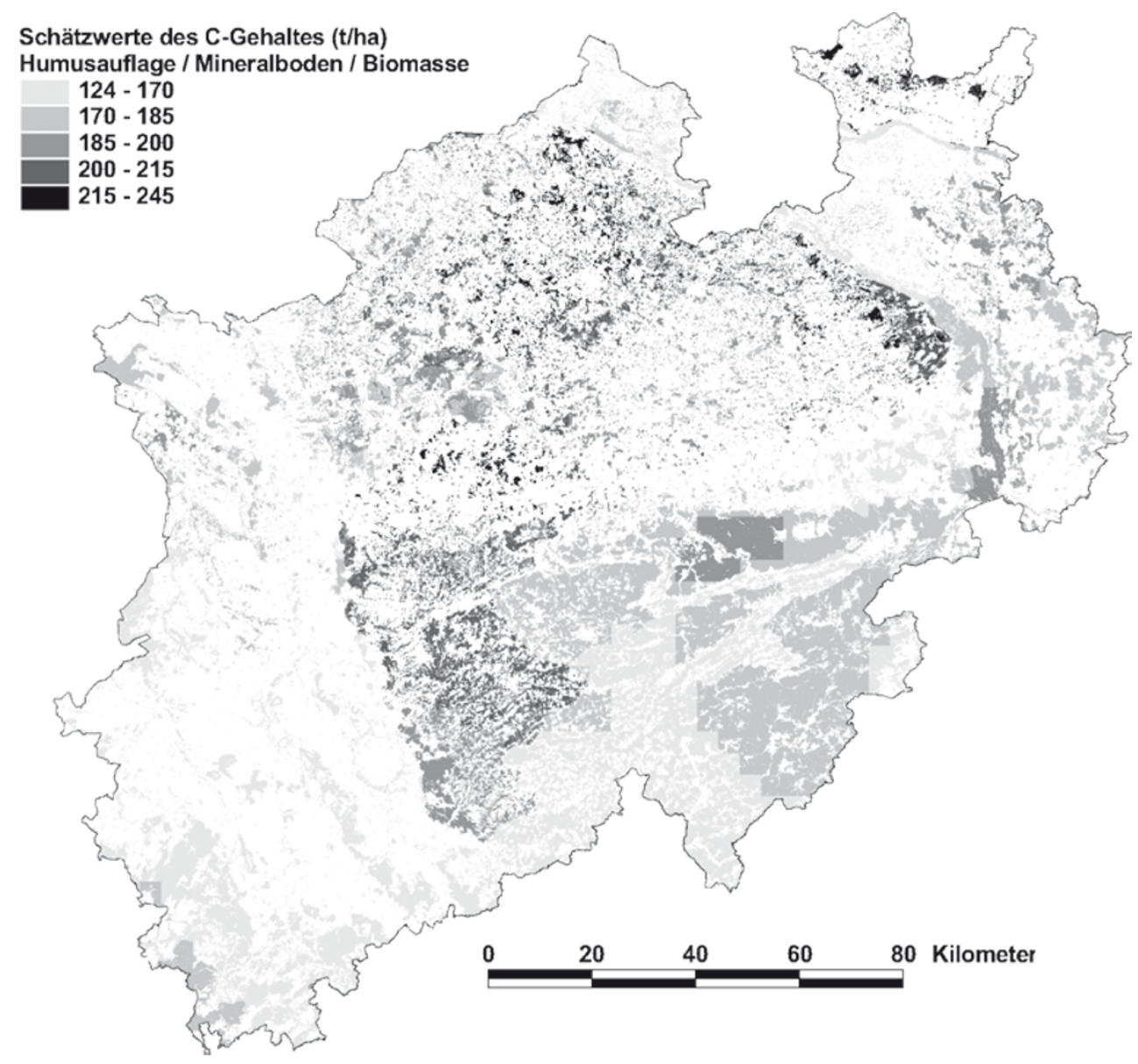

\section{Diskussion}

Das methodische Konzept dieser Untersuchung fußt auf einer Kombination von geostatistischen und multivariatstatistischen Verfahren. Zuerst wurde versucht, eine flächenhafte Abschätzung der Kohlenstoffvorräte mit Variogrammanalyse und Kriging durchzuführen. Nur für die Vorräte in der Humusauflage konnte eine räumliche Autokorrelation nachgewiesen werden. Die Variogrammanalysen wurden jeweils sowohl mit den unveränderten wie auch mit lognormal transformierten Daten durchgeführt. Auch erfolgte die Analyse deterministischer Trends mittels polynomischer Funktionen erster und zweiter Ordnung, gefolgt von einer variogrammanalytischen Überprüfung der Residuen. Trotzdem konnten für die oberirdische Biomasse sowie für den Mineralboden keine validen Variogrammmodelle abgeleitet werden. Die flächenhafte Abschätzung der Kohlenstoffvorräte hierfür gelang jedoch mit einer regelbasierten prädiktiven Kartierung der Kohlenstoffvorräte unter Verwendung der Entscheidungsbaumverfahren CART und CHAID. Der in der vorgestellten Untersuchung entwickelte Ansatz ist geeignet, forstliche und bodenkundliche Inventurdaten für eine großräumige Regionalisierung der Koh- lenstoffvorräte in den Wäldern Nordrhein-Westfalens zu nutzen.

Für die Wälder von Nordrhein-Westfalen konnten im oberirdischen Derb- und Totholz ca. 59 Mio. Tonnen Kohlenstoff (67t/ha), für die Humusauflage ca. 17 Mio. Tonnen Kohlenstoff (19,3t/ha) und für den Mineralboden (bis max. $2 \mathrm{~m}$ Tiefe) ca. 80 Mio. Tonnen Kohlenstoff $(90,7 \mathrm{t} /$ ha) ermittelt und räumlich differenziert werden. Daraus ergibt sich für die Wälder von NRW ein C-Vorrat von ca. 156 Mio. Tonnen Kohlenstoff für diese drei Kompartimente. Dies entspricht einem durchschnittlichen Wert von ca. $177 \mathrm{t} \mathrm{C} / \mathrm{ha}$.

Die in dieser Studie errechneten C-Gehalte sollen im Folgenden mit Werte aus weiteren Untersuchungen zur Speicherung von Kohlenstoff in Wäldern verglichen werden. Joosten und Schulte (2001) geben in einer überschlägigen Gesamtbilanz von NRW an, dass in den Wälder etwa 183 Mio. Tonnen Kohlenstoff gespeichert sind. Der durchschnittliche C-Gehalt pro Hektar Waldfläche liegt demnach bei 206,5 t. In die Bilanz von Joosten und Schulte (2001) fließen allerdings auch die C-Gehalte der Wurzelmasse (14,6t/ha, pauschale Schätzung: $20 \%$ der oberirdischen Masse), die Kraut- und Strauchschicht 
(1,1 t/ha), die Holzerntereste (3,6t/ha) und das Baumreisig (zusammen mit dem Derbholz 76,8 t/ha) ein. Der von Joosten und Schulte (2001) ohne Methodenhinweis angegebene Kohlenstoffgehalt in der Humusauflage liegt mit $16,9 \mathrm{t} / \mathrm{ha}$ (15 Mio. Tonnen) um 2,4t/ha niedriger als der in der vorliegenden Studie errechnete C-Gehalt. Baritz und Strich (2000) errechneten einen durchschnittlichen Kohlenstoffgehalt in der Humusauflage für die Wälder von Deutschland von 20,7 t/ha (Baritz 1996). Damit liegt dieser bundesdeutsche Durchschnittswert etwas höher als in Humusauflage der nordrhein-westfälischen Wälder. Wirth et al. (2004) konnten in einer Studie über die Wälder des Landes Thüringen nachweisen, dass im Humus durchschnittlich $27,7 \pm 8,1 \mathrm{t} \mathrm{C} /$ ha gebunden sind. Auffällig ist der große Unterschied zwischen Nadelwald und Laubwald: Unter Nadelwald wurden etwa dreifach höhere Werte als unter Laubwald ermittelt; 34,0 t/ha bzw. 11,3t/ha. Für den Mineralboden bis zu einer maximalen Tiefe von $90 \mathrm{~cm}$ der Wälder von Deutschland berechneten Baritz und Strich (2000) einen Durchschnittswert von 87,9t C/ha. Damit nähert sich dieser durchschnittliche Wert dem in dieser Studie ermittelten Wert von 90,7 t C/ha an. Es muss dabei berücksichtigt werden, dass in den vorliegenden Untersuchungen die C-Gehalte des Mineralbodens bis in eine maximale Bodentiefe von $2 \mathrm{~m}$ einflossen. Auf die große Schwankungsbreite der Kohlenstoffgehalte im Mineralboden weisen Baritz et al. (2005a,b) hin: Böden aus kalkhaltigem Ausgangsgestein weisen mit bis zu $115 \mathrm{t} / \mathrm{ha}$ die höchsten C-Gehalte auf, während die stauwassergeprägten Pseudogleye mit $47 \mathrm{t} / \mathrm{ha}$ die niedrigsten C-Gehalte aufweisen. Joosten und Schulte (2001) geben den C-Vorrat des Mineralbodens unter Wald in NRW mit 83 Mio. Tonnen an. Der sich daraus ergebende durchschnittliche Wert von $93,7 \mathrm{t} /$ ha liegt damit um $3 \mathrm{t} / \mathrm{ha}$ höher als der in dieser Studie ermittelte. Insgesamt lässt sich sagen, dass die hier bilanzierten Werte über den Kohlenstoffvorrat im Derbund Totholz, in der Humusauflage und im Mineralboden plausibel sind.

\section{Empfehlungen und Ausblick}

Inwieweit mit dem von Zirlewagen (2003) gewählten Regression Kriging, einer Kombination aus Regressionsanalyse und geostatistischer Flächenschätzung, die Befunde dieser Studie bestätigt werden können, wird in einer weiteren Studie überprüft. Eine baumartenspezifische Auswertung der vorhandenen Datensätze könnte einen weiteren Erkenntnisgewinn hinsichtlich der Kohlenstoffvorräte im Boden und der Biomasse erbringen. Eine solche Studie könnte in Hinblick auf die Reduktion von Treibhausgasen wichtige Hinweise für die zukünftige Waldbewirtschaftung liefern. Zusätzlich sollte berechnet werden, wie sich die Kohlenstoffspeicherung unter veränderten Klimabedingungen entwickeln wird. Hierfür sollten sowohl statistische Verfahren als auch die dynamische Modellierung mit dem Modellsystem WASMOD eingesetzt werden (Rinker et al. 2007). Sinnvoll wäre es auch, multivariate Kriging-Methoden, wie Co-Kriging und Simple-UpdateKriging, auf die vorhandenen Datensätze anzuwenden. Ferner sollte der entwickelte Ansatz anhand bundesweit vorliegender Daten und unter Nutzung der Daten der von Hornsmann et al. (2008) vorgelegten landschaftsökologischen Raumgliederung Europas für das Gebiet der EU validiert werden. Das Projekt zeigt, dass die Verknüpfung vorhandener Umweltdaten die Bearbeitung aktueller Fragen wie die der Kohlenstoffspeicherung in Wäldern wirkungsvoll unterstützen kann. Dies könnte und sollte durch den Einsatz internetbasierter Geoinformationssysteme WebGIS - weiter verbessert werden (Aden et al. 2008; Kleppin et al. 2008).

Danksagung Für die Überlassung der Daten der Landeswaldinventur Nordrhein-Westfalen, der Bodenzustandserhebung und zusätzlicher Flächeninformationen sowie für fachlich-wissenschaftliche Anregungen bedanken sich die Autoren beim Landesamt für Natur, Umwelt und Verbraucherschutz NRW (LANUV).

\section{Literatur}

AKS (Arbeitsgemeinschaft Standortskartierung in der Arbeitsgemeinschaft Forsteinrichtung (2005) Waldökologische Naturräume. Forstliche Wuchsgebiete und Wuchsbezirke in der Bundesrepublik Deutschland. Mitt Ver Forstl Standortskunde Forstpflanzenzüchtung 43

Aden C, Keppin L, Schmidt G, Schröder W (2008) WaldIS - a webbased reference data system for the forests in Germany. In: Car A, Griesebner G, Strobl J (eds) Geospatial Crossroads @ GI_Forum '08: Proceedings of the Geoinformatics Forum Salzburg. Wichmann, Heidelberg, pp 1-10

Baltes-Götz B (2004) Entscheidungsbaumanlyse mit AnswerTree 3.1. http://www.uni-trier.de/urt/user/baltes/docs/at/v31/at31.pdf Abrufdatum: März 2009

Baritz R (1996) Kohlenstoffvorräte der Waldböden Deutschlands, Teil I Kohlenstoffinventar. Reports Institute for Forest Ecology and Forest Inventory 1/1996, Federal Research Centre for Forestry and Forest Products, Hamburg, $55 \mathrm{~S}+$ annex

Baritz R, Strich S (2000) Forests and the national greenhouse gas inventory of Germany. Biotechnol Agron Soc Environ 4:267271

Baritz R, Rovira P, Romanya J, Erlandson M, Van Ranst E, Zirlewagen D, Jandl R, Seufert G, Olsson G (2005a) Multi-source inventory methods for quantifying carbon stocks and stock changes in European forests. CarboInvent: Validated, representative soil carbon profiles under forest vegetation. Part I Final Report D3.1, Doc. No WP3-D3.1-RUG, Issue/Rev. 2.0, Graz

Baritz R, Van Ranst E, Seufert G (2005b) Multi-source inventory methods for quantifying carbon stocks and stock changes in European forests. CarboInvent: Soil carbon default values relevant for evaluations of the carbon status of forest soils in Europe. Final report WP3-D3.2, Issue/Rev-No. 1.0, Graz

Beinhauer R (1999) Energieflüsse I. Agrarmeteorologische und bioklimatologische Prozesse. In: Fränzle O, Müller F, Schröder W 
(Hrsg) Handbuch der Umweltwissenschaften, 4. Erg. Lfg. ecomed, Landsberg, Kap. IV-2.1.1:1-14

BMELF (Bundesministerium für Ernährung, Landwirtschaft und Forsten) (1994) Bundesweite Bodenzustandserhebung im Wald (BZE). Arbeitsanleitung, 2. Aufl. Bonn

Breimann L, Friedmann JH, Olshen RA, Stone CJ (1984) Classification and regression trees. Wadsworth, Belmont, CA

EEA (European Environment Agency) (2004) Impacts of Europe's changing climate. An indicator-based assessment. EEA Report 2/2004. Copenhagen

Fränzle O, Killisch WF (1980) Aufschlüsselung des Informationsgehaltes umweltrelevanter, flächenbezogener Strukturdaten. Umweltforschungsplan des Bundesministers des Innern, ÖkologieForschungsbericht 10104 035, S VII, 436, Bonn

Freer-Smith P, Evans S, Morgan G, Ilvesniemi H, Finer L, Moffat A, Derome J, Humphrey J, Laine J, Michalak R, Pawlaczyk P (2004) Final Report for EU Contract Development and review concerning Forest Focus. Metla Forest Research, Helsinki

Hornsmann I, Schmidt G, Schröder W (2008) Berechnung einer landschaftsökologischen Raumgliederung Europas. Umweltwiss Schadst Forsch 20:25-35

IPCC (Intergovernmental Panel on Climate Change) (2002) Climate change and biodiversity. IPCC Technical Paper V. Geneva

IPCC (2007) The physical science basis. Contribution of Working Group I to the Fourth Assessment Report of the IPCC. Cambridge University Press, Cambridge

Isaaks EH, Srivastava RM (1989) An introduction to applied geostatistiks. Oxford University Press, Oxford

Joosten R, Schulte A (2001) Kohlenstoff- und Energieholzpotenziale der Wälder in Nordrhein-Westfalen. In: Schulte A, Böswald K, Joosten R (Hrsg) Weltforstwirtschaft nach Kyoto. Wald und Holz als Kohlenstoffspeicher und regenerativer Energieträger. Berichte aus der Holz- und Forstwirtschaft. Shaker, Aachen, S 123-136

Kass GV (1980) An exploratory technique for investigating large quantities of cate-gorial data. Appl Stat 29:199-127

Kleppin L, Schröder W, Pesch R, Schmidt G (2008) Entwicklung und Erprobung einer Metadaten- und WebGIS-Applikation für das Expositionsmonitoring mit Moosen in Deutschland. Ein Beitrag zum LTER-Netzwerk. Umweltwiss Schadst Forsch 20:38-48

Knigge W, Schulz H (1966) Grundriss der Forstbenutzung. Entstehung, Eigenschaften, Verwertung und Verwendung des Holzes und anderer Forstprodukte. Parey, Hamburg Berlin

Krige DG (1951) A statistical approach to some basic mine valuation problems on the witwatersrand. J Chem Mineral Soc S Afr $52: 119-139$
Matheron G (1965) Les variables regionalisées et leur estimation. Masson, Paris

Meynen E (Hrsg) (1953-1962) Handbuch der naturräumlichen Gliederung Deutschlands, Teil 1. Selbstverlag der Bundesanstalt für Landeskunde, Remagen

Meynen E (Hrsg) (1959-1962): Handbuch der naturräumlichen Gliederung Deutschlands, Teil 2. Selbstverlag der Bundesanstalt für Landeskunde, Remagen

Pesch R, Jerosch K, Schlüter M, Schröder W (2008) Using decision trees to predict benthic communities within and near the German Exclusive Economic Zone (EEZ) of the North Sea. Environ Monit Assess 136:313-325

Rinker A, Deunert F, Schröder W (2007) Modellierung der Auswirkungen des Klimawandels auf Phosphat- und Stickstoff-Austräge schleswig-holsteinischer Böden. In: Strobl J, Blaschke Th, Griesebner G (Hrsg) Angewandte Geoinformatik 2007. Beiträge zum 19. Agit-Symposium. Wichmann, Heidelberg, S 621-630

Schröder W (1989) Ökosystemare und statistische Untersuchungen zu Waldschäden in Nordrhein-Westfalen: Methodenkritische Ansätze zur Operationalisierung einer wissenschaftstheoretisch begründeten Konzeption. Diss Univ Kiel

Schröder W, Schmidt G (2001) Raumgliederung für die Ökologische Umweltbeobachtung des Bundes und der Länder. Umweltwiss Schadst Forsch 12:237-243

Schröder W, Schmidt G, Hornsmann I (2006) Landschaftsökologische Raumgliederung Deutschlands. In: Fränzle O, Müller F, Schröder W (Hg) (1997-2004): Handbuch der Umweltwissenschaften. Grundlagen und Anwendungen der Ökosystemforschung. Kap. V-1.9, 16. Erg. Lfg., Landsberg am Lech München Zürich, S 1-101

Schröder W, Englert C, Schmidt G (2007) Phänologische Änderungen bei Obstbäumen und anderen Pflanzen sowie weitere mögliche Folgen des Klimawandels für die Landwirtschaft. Land Info $5: 15-28$

Webster R, Oliver MA (2001) Geostatistics for environmental scientists. Wiley, Chichester

Wirth C, Schulze ED, Schwalbe G, Tomczyk S, Weber G, Weller E (2004) Dynamik der Kohlenstoffvorräte in den Wäldern Thüringens. Abschlussbericht zur 1. Phase des BMBF-Projektes „Modelluntersuchung zur Umsetzung des Kyoto-Protokolls“. Mitt Thüringer Landesanst Wald Jagd Fischerei, Jena Gotha, 23:1-308

Zierdt M 1997 Umweltmonitoring mit natürlichen Indikatoren. Springer, Berlin Heidelberg New York, S 1-215

Zirlewagen D (2003) Regionalisierung bodenchemischer Eigenschaften in topographisch stark gegliederten Waldlandschaften. Freiburger Forstliche Forschung 19, Freiburg 